Casos Clínicos

Arch. Esp. Urol. 2010; 63 (1): 78-84

\section{LINFOMA DE CÉLULAS T EN EL TESTÍCULO. PRESENTACIÓN DE 2 CASOS CLÍNICOS Y REVISIÓN DE LA LITERATURA}

\author{
Oscar Alberto Messa Botero y Julio Alexander Díaz \\ Pérez?
}

Grupo de Patología del Instituto Nacional de Cancerología. Bogotá. Colombia.

'Fundación para el Avance de la Anatomía Patológica. Citología y Clínica Molecular FAPCIM. Bucaramanga.

Universidad de Santander UDES. Bucaramanga. Colombia.

Resumen.- OBJETIVO: Presentar dos casos clínicos de linfoma de células T con compromiso testicular diagnosticados y realizar una discusión sobre este tema.

MÉTODOS: Caso 1. Hombre de 42 años, con masa y dolor testicular derecho, de 2 meses de evolución. Cuya ecografía testicular evidencio aumento difuso del tamaño del testículo, con alteración de la ecogenicidad. Fue llevado a orquidectomía, diagnosticando con estudios de histopatología e inmunohistoquímica un linfoma de células $T$ periférico inespecífico. Caso 2. Hombre de 44 años quien consulta por aumento de volumen del testículo izquierdo de 3 meses de evolución, con lesiones maculares cutáneas en muslo y espalda de color pardo rojizo, asociadas, con posterior compromiso del testículo derecho. La ecografía

\section{CORRESPONDENCIA}

Julio Alexander Díaz Pérez

Fundación para el Avance de la Anatomía Patológica, Citología y Clínica Molecular FAPCIM. Bucaramanga. (Colombia).

pat_uis@yahoo.com

Trabajo recibido: 6 de julio 2009. muestro testículos aumentados de tamaño, con alteración difusa de su ecogenicidad. Por lo anterior, realizan orquidectomía izquierda. Con el estudio histopatológico e inmunofenotípico, diagnóstico un linfoma no Hodgkin, T periférico no especificado.

CONCLUSIÓN: Los linfomas de células T pueden comprometer los testículos en casos infrecuentes, que merecen especial atención debido al pobre pronóstico de esta enfermedad, y a la necesidad de realizar un adecuado diagnóstico que puede ayudar a implementar mejores estrategias terapéuticas.

Palabras clave: Linfoma de células T. Testículo. Serie de Casos. Revisión de la literatura.

Summary.- OBJECTIVE: To present two clinical cases of testicular T cell lymphomas and perform a review of this condition.

METHODS/RESULTS: Case 1. Forty-two year old male presenting with painful mass in the right testicle which appeared 2 months earlier. Testicular ultrasound showed diffuse increase in size of the testicle, with alteration in its echogenicity. The patient underwent orchidectomy, and based on histopathological and immunohistochemical tests, a peripheral nonspecific T cell lymphoma was diagnosed. Case 2. Forty-four year old male who presents with a 3 month history of left testicular enlargement without pain associated with reddish-brown macular lesions in the thigh and back and subsequent involvement of the right side of the scrotum. The ultrasonography showed testicles with increased size, with echogenicity altered diffusely. Left orchidectomy was performed and sent for histopathological and Immunophenotypic study which revealed a nonHodgkin peripheral T cell lymphoma.

CONCLUSION: T cell lymphomas involve the testis infrequently, which deserve special attention because of the poor prognosis and the need to make an appropriate diagnosis which could lead to a better therapeutic strategy.

Keywords: T Cell Lymphoma. Testis. Cases report. Review.

\section{INTRODUCCIÓN}

Los tumores testiculares son en general poco frecuentes, ellos representan solo el 1 al $2 \%$ de los tumores que afectan a los hombres, son más frecuentes entre los 20 y los 35 años de edad $(1,2)$, donde se observa una clara predominancia por tumores de origen germinal, por lo anterior, los tumores de este linaje son los más frecuentes en forma global representando entre el 85 al $90.4 \%$ de las neoplasias primarias de los testículos (1). Los linfomas testiculares son mucho menos frecuentes, 
ellos se encuentran solo en el al $9 \%$ de los tumores testiculares, se observan con una incidencia de 0.26 casos por 100.000 personas-año, y se presentan con mayor frecuencia en pacientes mayores de 60 años, grupo de edad donde llegan a constituir el mayor porcentaje de neoplasias testiculares (2). Los linfomas testiculares son generalmente de células $B$, entre los que se destaca el linfoma difuso de células B grandes, el cual es el más común subtipo observado, pero también, se han informado muy pocos casos de linfomas de células T (3).

Los linfomas que comprometen los testículos, son en general linfomas no Hodgkin, que poseen características biológicas inmunofenotipicas, clínicas y de comportamiento particulares, que permiten su reconocimiento, a pesar de su baja presentación $(1,4)$; de sus variantes tiene peculiar importancia el estudio de los linfomas de células $T$, ya que a pesar de su extraordinaria baja frecuencia, son generalmente letales, con una sobrevida media de 12 a 24 meses (5). Por lo anterior, se trazo como objetivo describir dos casos clínicos de linfoma de células $T$ con compromiso testicular diagnosticados en el Instituto Nacional de Cancerología de Colombia y realizar una discusión sobre este tema.

\section{CASOS CLÍNICOS}

\section{Caso 1}

Hombre de 42 años, quien consultó por masa y dolor testicular derecho, de 2 meses de evolución, que aumentó en forma progresiva. Niega antecedentes patológicos y otra sintomatología. La ecografía testicular evidencio aumento difuso del tamaño del testículo derecho, el cual mide $6.2 \times 5.8 \times 4.2 \mathrm{~cm}$, con alteración difusa de la ecogenicidad del mismo. Por lo cual, fue llevado a orquidectomia encontrando testículo aumentado de tamaño, cauchoso, blanquecino. En el estudio histopatologico se evidencio la infiltración difusa del parénquima testicular por una neoplasia maligna hematolinfoide compuesta por células de pequeño tamaño, irregulares, de citoplasma escaso, las cuales presentaban angiotropismo, con áreas de necrosis asociadas. Las células tumorales
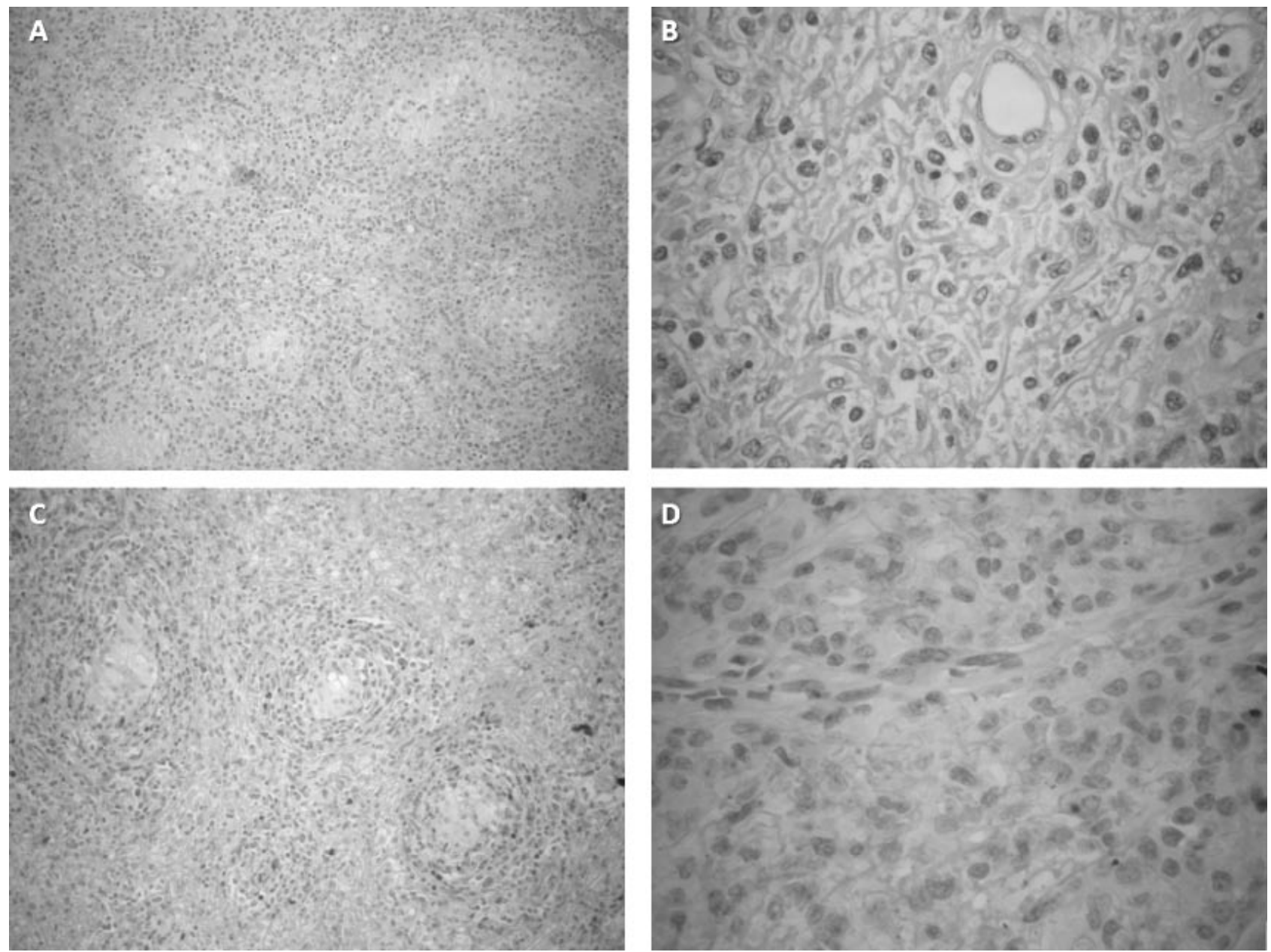

FIGURA 1. Se reconoció la presencia de un infiltrado difuso de células linfoides neoplasicas las cuales distorsionaban por completo la histología testicular, en los que solo se aprecian esbozos de conductos seminíferos (A y B, HE 4x y 14x), estas células expresaban bol 2 (C, 10x) y eran negativas para CD56 (D, 40c). 
fueron positivas para $\mathrm{ACL}$ y $\mathrm{CD} 3$ con $\mathrm{Ki67}$ del $90 \%$ y negativas para TdT, CD30, ALK, bcl2, bcl6 y CD10. (Figura 1). Con lo anterior se realizo el diagnostico de Linfoma no Hodgkin T periférico no especificado.

\section{Caso 2}

Hombre de 44 años quien consulta por aumento de volumen del testículo izquierdo de 3 meses de evolución, presentó además lesiones maculares cutáneas en muslo y espalda de color pardo rojizo, pérdida de 2 $\mathrm{Kg}$ de peso en el último mes, fiebre no cuantificada, diaforesis nocturna, sin otros síntomas asociados. Posteriormente presenta compromiso del testículo derecho. Refiere antecedente de safenectomia en pierna derecha por insuficiencia vascular y padre con hepatocarcinoma. Al examen físico se observan testículos aumentados de volumen. La ecografía muestra testículos aumentados de tamaño, testículo derecho de $5.4 \times 3.8 \times 3.9 \mathrm{~cm}$, con presencia de masa solida, hipoecoica, heterogénea, de $4 \mathrm{~cm}$. de diámetro y testículo izquierdo de $6.4 \times 2.9 \times$
$3.8 \mathrm{~cm}$., con alteración difusa de su ecogenicidad, la cual luce disminuida, observándo escaso parénquima testicular respetado hacia su polo inferior, con aumento del flujo de baja resistencia lo que sugiere un proceso linfoproliferativo. La TAC de abdomen evidenció masas testiculares bilaterales, y múltiples adenopatías retroperitoneales y masas que podrían corresponder a conglomerado ganglionar en fosas adrenales bilaterales. La BHCG fue menor de $5 \mathrm{UI} / \mathrm{L}$, la LDH fue de 1,29, la AFP fue de 2,2 y el examen de ELISA para VIH fue negativo en dos oportunidades. Por lo anterior, realizan orquidectomia izquierda encontrando, un testículo aumentado de tamaño, de $6 \times 3.5 \times 3.5 \mathrm{~cm}$ de superficie parda el cual al corte presenta una lesión amarillenta mal definida, de $3 \times 2 \times 2 \mathrm{~cm}$ que ocupaba el polo superior.

El estudio histopatológico mostró extenso compromiso por una neoplasia hematolinfoide compuesta por células irregulares de núcleos pleomórficos con mitosis, angioinvasión y compromiso de túbulos seminiferos. El estudio inmunofenotipico fue positivo para $\mathrm{ACL}, \mathrm{CD} 45 \mathrm{RO}$
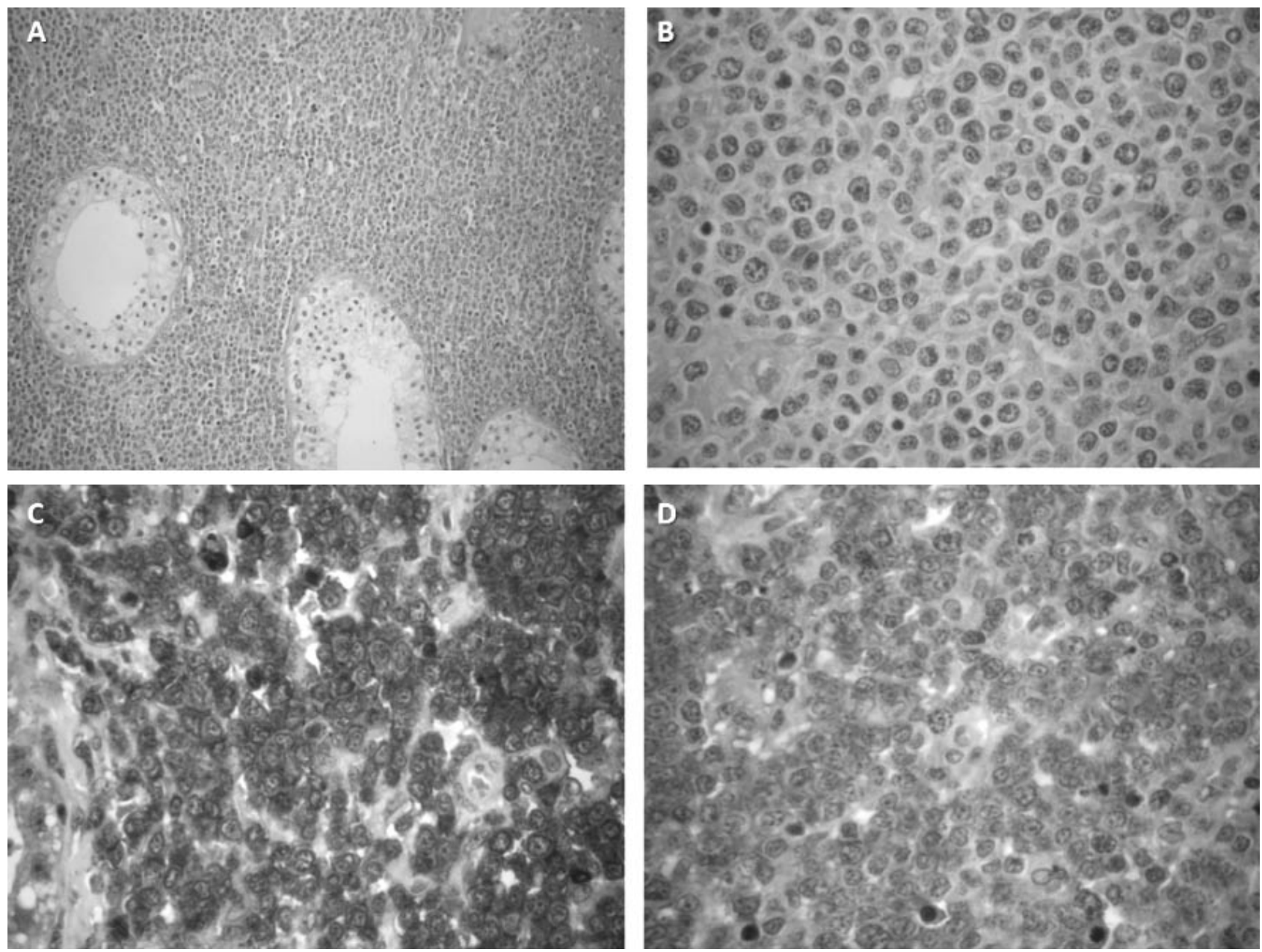

FIGURA 2. En el caso 2 se observo la presencia de gran cantidad de células linfoides neoplasicas distribuidas en forma difusa, que distorsionan la arquitectura testicular lesionando los conductos seminíferos $(A, 4 x)$, de tamaño predominantemente pequeño, irregulares, con escaso citoplasma $(B, 40 x)$, las cuales fueron positivas para CD3 $(C, 40 x)$ y CD56 (D, 
(UCHL-1), CD3 y Bcl2, con débil expresión de CD43, CD4 y CD2, alto índice proliferación (ki67 del 50\%), y pérdida de la expresión de CD5, CD7, CD56, CD20, CD8, CD 10, CD57, Bcl6, fascin, CD23, PLAP, AE 1AE3, CD30, ALK, TdT, S100 y MPOX (Figura 2). Con lo anterior se realizó el diagnóstico de linfoma no Hodgkin, T periférico no especificado. Los estudios de extensión en médula ósea y líquido cefalorraquídeo fueron negativos. El paciente fue manejado con quimioterapia $\mathrm{CHOP}$ y quimioterapia intratecal con MTX, falleciendo luego de 6 meses de seguimiento.

\section{Estrategia de búsqueda de la literatura}

Se realizó una búsqueda estructurada de la literatura, con base en la metodología Cochrane, en las bases de datos Medline, Imbiomed y Scielo, desde Enero del año 1950 a Marzo de 2009. Se utilizaron como palabras clave "Lymphoma", "Testis" y "T cell". Se limito la búsqueda a artículos originales escritos en idioma inglés y español, la metodología empleada se muestra en la Tabla 2. De los artículos encontrados, se seleccionaron los considerados relevantes por los autores para realizar a discusión del tema.

\section{DISCUSIÓN}

Los linfomas testiculares fueron inicialmente informados por Malassez en 1866 y fueron posteriormente definidos como una entidad clínica particular por Curling al final del siglo XIX (1). Estas neoplasias representan únicamente el 1 al $4 \%$ de todos los linfomas no Hodgkin extranodales y el $5 \%$ (entre el 1 y el $9 \%$ ) de todas las neoplasias malignas testiculares, pero a pesar de su baja frecuencia son las neoplasias gonadales más comunes en pacientes mayores de 60 años de edad $(1,2)$. El compromiso de los testículos por un linfoma, es en la mayoría de los casos de origen secundario, hecho que se observa en más del $4 \%$ de los linfomas tipo Burkitt, así como en otros tipos de linfoma $(5,6)$, siendo pocos los casos informados de compromiso testicular primario $(3,4,7)$. Hasta el momento la etiología de los linfomas testiculares primarios es desconocida, pero se han documentado algunos factores predisponentes en informes anecdóticos como: trauma, orquitis crónica, criptorquidia o filariasis (5); también al igual a lo observado con otros linfomas extragonadales, los linfomas testiculares se observan más frecuentemente en los pacientes afectados por el Síndrome de Inmuno-Deficiencia Adquirida (SIDA), en los cuales llegan a ser responsables de hasta el $25 \%$ de los tumores testiculares observados (8). Además en los pacientes con SIDA esta neoplasia se caracteriza por una aparición a una edad más joven, presentar variedades histopatologicas más agresivas y tener un peor pronóstico (5).

La manifestación clínica más común es la presencia de dolor testicular, desencadenado con la palpación o espontánea, de semanas a meses de evolución, con aumento de volumen y de consistencia testicular generalmente unilateral; aunque también se puede presentar como una masa testicular indolora $(1,2)$. Además se debe tener en cuenta en la evaluación clínica que el linfoma es el tumor bilateral más común de los testículos, en el cual también, se ha encontrado compromiso del testículo contralateral en los casos de linfoma unilateral hasta en el $35 \%$ de los casos. Estas neoplasias pueden comprometer el epidídimo, el cordón espermático, piel escrotal y los ganglios linfáticos retroperitoneales; y pueden diseminarse a distancia a múltiples sitios extranodales como el testículo contralateral, sistema nervioso central $(6-16 \%)$, piel $(0-35 \%)$, anillo de Waldeyer $(5 \%)$, pulmón, pleura y tejidos blandos. El compromiso secundario de los testículos en pacientes con linfoma es más común que el hallazgo de un linfoma testicular primario $(3,6)$. Para separar estas dos entidades se ha establecido como regla, la presencia de una masa testicular, sin evidencia de compromiso por la enfermedad en otras

TABLA I. ESTRATEGIA DE BÚSQUEDA DE LA LITERATURA, SE UTILIZAN DESCRIPTORES MESH PARA LA BASE DE DATOS MEDLINE, PARA LAS DEMÁS BASES DE DATOS LA BÚSQUEDA SE REALIZO EN FORMA ANÁLOGA.

\begin{tabular}{|c|l|c|}
\hline Paso & Descriptor (Palabra Clave) & Numero de Citaciones \\
\hline 1 & T Cell Lymphoma & 25975 \\
\hline 2 & Testis & 74782 \\
\hline 3 & $\# 1$ AND \#2 & 87 \\
\hline 4 & Limits & 56 \\
\hline
\end{tabular}


áreas del cuerpo, para ser definido como linfoma testicular primario $(1,3)$. A pesar de lo anterior, esta distinción es muchos casos es imposible, debido a que un linfoma testicular primario en estadio IV es virtualmente indistinguible de un linfoma nodal avanzado con compromiso testicular y viceversa.

Como todas las neoplasias hematolinfoides en el estudio de los linfomas testiculares, se debe realizar un examen hematológico y bioquímico completo, en los cuales generalmente la LDH está elevada, correlacionándose directamente con la actividad tumoral $(2,6)$. Otros marcadores tumorales como la AFP, B-HCG, CEA son normales. Además se deben realizar estudios de extensión para estadificar correctamente la enfermedad que deben incluir estudios imagenológicos de tórax, abdomen y cráneo, además de un estudio de biopsia de medula ósea.

TABLA II. CARACTERÍSTICAS DEL PANEL DE INMUNOHISTOQUIMICA EMPLEADO EN EL ESTUDIO DEL CASO.

\begin{tabular}{|c|c|c|c|}
\hline Marcador & Clon & Dilución & Casa Comercial \\
\hline $\mathrm{ACL}$ & $2 B 11+P D 7 / 26$ & $1: 100$ & DakoCytomation \\
\hline AE IAE3 & $\mathrm{AE} 1 / \mathrm{AE} 3$ & $1: 100$ & DakoCytomation \\
\hline ALK/ p80 Ab-1 & $5 \mathrm{~A} 4$ & $1: 40$ & Thermo Scientific \\
\hline $\mathrm{Bcl} 2$ & $\mathrm{Bcl} 2 / 100 / \mathrm{D} 5$ & $1: 50$ & Novocastra \\
\hline Bcl6 & PIFG & $1: 50$ & ZYMED \\
\hline CD2 & $A B 75$ & $1: 25$ & BioGenex \\
\hline CD3 & UCHT-1 & $1: 50$ & DakoCytomation \\
\hline CD4 & $1 F 6$ & $1: 50$ & ZYMED \\
\hline CD5 & $4 C 7$ & $1: 50$ & ZYMED \\
\hline CD7 & 272 & $1: 20$ & BioGenex \\
\hline CD8 & $1 \mathrm{~A} 5$ & $1: 50$ & ZYMED \\
\hline CD10 & $56 C 6$ & $1: 60$ & Thermo Scientific \\
\hline CD20 & L26 & $1: 50$ & ZYMED \\
\hline CD23 & MHM6 & $1: 100$ & DakoCytomation \\
\hline CD30 & $1 G 12$ & $1: 50$ & Novocastra \\
\hline CD43 & DF-TI & $1: 50$ & Thermo Scientific \\
\hline CD45RO & UCHL-1 & $1: 50$ & DakoCytomation \\
\hline CD56 & BC56C04 & $1: 50$ & Biocare Medical \\
\hline CD57 & 123C3.D5 & $1: 50$ & Thermo Scientific \\
\hline ki67 & MIB- 1 & $1: 50$ & DakoCytomation \\
\hline Fascin & $55 k-2$ & $1: 50$ & DakoCytomation \\
\hline MPOX & & $1: 300$ & DakoCytomation \\
\hline PLAP & 8 A9 & $1: 200$ & DakoCytomation \\
\hline$S 100$ & & $1: 50$ & ZYMED \\
\hline $\mathrm{TdT}$ & SEN28 & $1: 30$ & Thermo Scientific \\
\hline
\end{tabular}


Ecográficamente, se puede observar afectación focal o difusa de áreas con disminución de la ecogenicidad, en testículos aumentados de tamaño (10). De manera puntual se han descrito casos de linfomas que ecográficamente, presentan un aumento de la ecogenicidad. El diagnóstico diferencial ecográfico debe realizarse con seminomas, orquitis crónica piógena, brucelosis testicular, tuberculosis testicular, rabdomiosarcoma y leucemia (3). La Tomografía Axial Computarizada (TAC) muestra lesiones con márgenes espiculados, al igual que las imágenes de resonancia magnética (11).

Los linfomas que pueden comprometer los testículos son un grupo muy heterogéneo, el tipo más común de linfoma testicular primario, es el linfoma de células grandes B difuso, el cual es el responsable de alrededor del 70 $90 \%$ de los casos informados. Los demás subtipos informados incluyen linfoma folicular, plasmocitoma, linfoma linfoblastico, linfoma Burkitt y linfoma Burkitt like, siendo muy infrecuente el hallazgo de linfomas de células $T$ $(2,12,13)$. El procedimiento diagnóstico de elección es la orquidectomía, ya que con ella se logra obtener la cantidad necesaria de tejido para realizar un apropiado diagnóstico, las biopsias guiadas por ultrasonido no son recomendadas, por el escaso material obtenido y la factibilidad de diseminación neoplásica. Un adecuado análisis con técnicas de inmunohistoquimica y citome-

TABLA III. COMPARACIÓN DE LOS PERFILES INMUNOFENOTIPICOS DE LOS CASOS ESTUDIADOS.

\begin{tabular}{|c|c|c|}
\hline Marcador & Caso 1 & Caso 2 \\
\hline ACL & Positivo & Positivo \\
\hline CD3 & Positivo & Positivo \\
\hline UCHL-1 & Positivo & Positivo \\
\hline Bcl2 & Negativo & Positivo \\
\hline CD56 & Positivo & Negativo \\
\hline CD10 & Negativo & Negativo \\
\hline CD23 & Negativo & Negativo \\
\hline CD20 & Negativo & Negativo \\
\hline CD30 & Negativo & Negativo \\
\hline ALK & Negativo & Negativo \\
\hline TdT & Negativo & Negativo \\
\hline Ki67 & $90 \%$ & $50 \%$ \\
\hline
\end{tabular}

tría de flujo permite en muchos casos establecer un diagnóstico adecuado $(1,14)$. La mayoría de los linfomas testiculares presentan un inmunofenotipo de células $B$. Otros subtipos histopatológicos observados son el linfoma de Burkitt, los linfomas Burkitt like, y muy raramente los linfomas de células T (15). Estos últimos, se caracterizan por su alta agresividad, con medias de sobrevida de 10 a 30 meses. Las características generales que en el estudio histopatológico con tinción de hematoxilina y eosina, se encuentran, en general, asociados a la presencia de un fenotipo $T$ son: polimorfismo celular, irregularidad de la membrana nuclear, infiltrado reactivo mixto acompañante que incluye eosinofilos, neutrofilos, histiocitos y linfoplasmocitos; adicionalmente es frecuente observar angioinvasion que ocasiona extensas áreas de necrosis asociada; y en ocasiones se encuentra un infiltrado difuso linfocitico con numerosos histiocitos de tipo epitelioide, que puede llegar a semejar al linfoma Hodking o al linfoplasmocítico; por lo anterior, siempre se deben realizar estudios de inmunohistoquímica o de citometría de flujo para su adecuada clasificación (14). En centros especializados se cuenta con estudios de monoclonalidad con la utilización de PCR para la evaluación de receptores de células $T$. El inmunofenotipo comúnmente observado muestra positividad para CD3, CD7, CD5 y CD2; raramente estos tumores también expresan marcadores de células $B$ como $C D 20$ y CD79a, al igual que marcadores de células NK CD16, CD56 y CD57 $(5,16,17)$. Los linfomas de células T que afectan al testículo en forma primaria son generalmente del los subtipos: linfoma de células $T$ del adulto, linfoma de células T/NK extranodal, linfoma de células $T$ angioinmunoblastico y linfoma de células $T$ periférico no especificado, el cual es el más comúnmente observado $(7,17)$.

En estos casos, no encontramos hallazgos histopatológicos que plantearan una neoplasia inmadura o de precursores (nucleos redondos-ovales, cromatinas vesiculares, conteo mitótico alto), adicionalmente el TdT fue negativo, lo que planteó una neoplasia T perifeca; se descartó linfoma anaplasico (ausencia de características morfológicas y de reactividad para CD30 y ALK), linfoma angioinmunoblástico (ausencia de criterios morfológicos y marcadores $T$ centrogerminales negativos CD10, Bcl6 y CD57, así como acúmulos de dendritas perivasculares con CD23) y linfoma T-NK de tipo nasal (falta de marcada angiocentricidad, necrosis y negatividad de CD56), los cuales son los linfomas T periféricos testiculares mas frecuentemente descritos. Por lo anterior, se clasificaron a estas neoplasias como linfomas no Hodking T periféricos no clasificado.

El diagnóstico diferencial incluye múltiples procesos neoplásicos, no neoplásicos, como lo son orquítis, epidimítis, torsión testicular, tumores germinales (seminoma, teratoma, tumor del saco vitelino y coriocarcinoma), tumores estromales, otros linfomas y lesiones metastásicas de diferentes orígenes que incluyen próstata, pulmón, melanoma y riñón $(2,5,6,18)$. 
El tratamiento de elección en los linfomas testiculares se basa en la quimioterapia y la cirugía $(6,9)$. La orquidectomía radical debe ser realizada tanto en estadios localizados como avanzados. El papel de la orquidectomía radical bilateral profiláctica es controvertido, aunque se justifica debido a la alta tasa de recidiva de esta enfermedad. La radioterapia aunque controvertida también es utilizada, en ella generalmente se usan dosis entre 2500 y 3500 cGY. Una pieza fundamental en el tratamiento es la utilización de ciclos cortos de quimioterapia (3 a 6 ciclos de CHOP o doxorubicin, ciclosfosfamida, vincristina y bleomocina), la cual evita la aparición de linfoma contralateral, aumenta el porcentaje de remisiones completas y la toxicidad es menor, en estadios tempranos y avanzados, con tasas de respuesta entre el 50 y el $80 \%(1,2,6,7,15)$.

El pronóstico de esta enfermedad es muy pobre, se ha encontrado que un peor desenlace se encuentra asociado a la avanzada edad de presentación, síntomas constitucionales, compromiso del cordón espermático, la variedad histopatológica, la presencia de invasión vascular y el bajo grado de esclerosis $(6,7,18)$.

\section{CONCLUSIÓN}

Los linfomas de células T pueden comprometer los testículos en casos infrecuentes, que merecen especial atención debido al pobre pronóstico de esta enfermedad, y a la necesidad de realizar un adecuado diagnóstico que puede ayudar a implementar mejores estrategias terapéuticas.

\section{BIBLIOGRAFÍA y LECTURAS RECOMENDADAS (*lectura de interés $y^{* *}$ lectura fundamental)}

**1. Vitolo U, Ferreri AJ, Zucca E. Primary testicular lymphoma. Crit Rev Oncol Hematol. 2008;65(2):183-9

2. Shahab N, Doll DC. Testicular lymphoma. Semin Oncol. 1999; 26:259-269.

*3. Zicherman JM, Weissman D, Gribbin C, Epstein R. Best cases from the AFIP: primary diffuse large Bcell lymphoma of the epididymis and testis. Radiographics 2005;25:243-8.

4. Al-Abbadi MA, Hattab EM, Tarawneh MS, Amr SS, Orazi A, Ulbright TM. Primary testicular diffuse large B-cell lymphoma belongs to the nongerminal center B-cell like subgroup: a study of 18 cases. Modern Pathol. 2006;19:1521-7.

5. Chávez Martínez VH, Landa Soler M, De la Sancha Mondragon LM. Linfoma no Hodgkin testicular primario de células $\mathrm{T}$ reporte de un caso y revi- sión de la literatura. Revista mexicana de urología 2007;67(1):65-9.

**6. Verma N, Lazarchick J, Gudena V, Turner J, Chaudhary UB. Testicular lymphoma: an update for clinicians. Am J Med Sci. 2008;336(4):336-41.

7. Chan JK, Tsang WY, Lau WH, Cheung MM, Ng WF, Yuen WC, et al. Aggressive T/natural killer cell lymphoma presenting as testicular tumor. Cancer. 1996 15;77(6):1198-205.

8. Lagrange JL, Ramaioli A, Theodore $\mathrm{CH}$, TerrierLacombe MJ, Beckendorf V, Biron P, et al. Radiation Therapy Group and the Genito-Urinary Group of the French Federation of Cancer Centres. NonHodgkin's lymphoma of the testis: a retrospective study of 84 patients treated in the French anticancer centres. Ann Oncol. 2001;12(9):1313-9.

*9. Vural F, Cagirgan S, Saydam G, Hekimgil M, Soyer NA, Tombuloglu M. Primary testicular lymphoma. J Natl Med Assoc. 2007;99(11):1277-82.

10. Bloom C, Hamilton P, Cassoff J, Pinsky M, Rosenbloom M, Sabloff B, et al. Scrotal ultrasonography: a pictorial essay. Can Assoc Radiol J. 1998;49(1):1220.

11. Saito W, Amanuma M, Tanaka J, Heshiki A. A case of testicular malignant lymphoma with extension to the epididymis and spermatic cord. Magn Reson Med Sci. 2002;1(1):59-63.

12. Lu D, Medeiros LJ, Eskenazi AE, Abruzzo LV. Primary follicular large cell lymphoma of the testis in a child. Arch Pathol Lab Med. 2001;125(4):551-4

13. Köksal Y, Yalçin B, Uner A, Akyüz C, Han U, Büyükpamukçu M. Primary testicular Burkitt lymphoma in a child. Pediatr Hematol Oncol. 2005;22(8):705-9.

14. Hasselblom S, Ridell B, Wedel H, Norrby K, Sender Baum M, Ekman T. Testicular lymphoma--a retrospective, population-based, clinical and immunohistochemical study. Acta Oncol. 2004;43(8):758-65.

15. Gómez García I, Rodríguez Patrón R, Sanz Mayayo E, Rodríguez Luna JM, Palmeiro Uriach A, Conde Someso S, et al. Primary testicular lymphoma. Report of a new case and review of the literature. Actas Urol Esp. 2004 Feb;28(2):141-6.

16. Kim YB, Chang SK, Yang WI, Hahn JS, Koom WS, Shim SJ, et al. Primary NK/T cell lymphoma of the testis. Acta Haematol 2003;109:95-100.

**17. Ornstein DL, Bifulco CB, Braddock DT, Howe JG. Histopathologic and molecular aspects of CD56+ natural killer/ T-cell lymphoma of the testis. Int J Surg Pathol. 2008;16(3):291-300.

18. Díaz Pérez GA, Pow-Sang Godoy M, Morante Deza C, Meza Montoya L, Destefano Urrutia V. Primary testicular lymphoma: experience at the Instituto Especializado de Enfermedades Neoplásicas, LimaPeru. Actas Urol Esp. 2009;33(2):149-53. 